\title{
Effective temperatures from the fluctuation-dissipation measure- ments in soft glassy materials
}

\author{
S. Jabbari-Farouji ${ }^{1,2, *}$ ，D. Mizuno ${ }^{3,4,5, *}$ ，D. Derks ${ }^{6}$, G.H. Wegdam ${ }^{1}$, F.C. MacKintosh ${ }^{2}$, C.F.
} SCHMIDT $^{3,5}$ AND D. BONN ${ }^{1,6}$

1 Van der Waals Zeeman Institut, Universiteit van Amsterdam, Valckenierstraat 65, 1018 XE, Amsterdam, The Netherlands

2 Theoretical Physics and Polymer Group, Department of Applied Physics, Technische Universiteit Eindhoven 5600MB Eindhoven, The Netherlands

3 Department of Physics and Astronomy, Vrije Universiteit Amsterdam, De Boelelaan 1081, 1081HV Amsterdam, The Netherlands

4 Organization for the Promotion of Advanced Research, Kyushu University, Higashi-ku, Hakozaki 6-10-1, 812-0054

Fukuoka, Japan

${ }^{5}$ Physikalisches Institut, Georg-August-Universität, Friedrich-Hund-Platz 1, 37077 Göttingen, Germany

6 Laboratoire de Physique Statistique, Ecole Normale Supérieure, 24, rue Lhomond, 75231 Paris Cedex 05, France

* Both authors have equally contributed to this work.

PACS $n n \cdot m m . x x-$ First pacs description

PACS $\mathrm{nn} \cdot \mathrm{mm} \cdot \mathrm{xx}-$ Second pacs description

PACS $\mathrm{nn} . \mathrm{mm} . \mathrm{xx}$ - Third pacs description

\begin{abstract}
We have investigated the validity of the fluctuation-dissipation theorem (FDT) and the applicability of the concept of effective temperature in a number of non-equilibrium soft glassy materials. Using a combination of passive and active microrheology to measure displacement fluctuations and the mechanical response function of probe particles embedded in the materials, we have directly tested the validity of the FDT. Our results show no violation of the FDT over several decades in frequency $\left(1-10^{4} \mathrm{~Hz}\right)$ for hard sphere colloidal glasses and colloidal glasses and gels of Laponite. We further extended the bandwidth of our measurements to lower frequencies (down to $0.1 \mathrm{~Hz}$ ) using video microscopy to measure the displacement fluctuations, again without finding any deviations from the FDT.
\end{abstract}

Introduction. - While the foundations of equilibrium statistical mechanics are well-established, nonequilibrium statistical physics is still being developed. One attempt in the direction of developing a statistical mechanical description of non-equilibrium, but slowly evolving systems is to extend the fluctuation dissipation theorem (FDT) to non-equilibrium situations [1]. The FDT relates the relaxation of spontaneous fluctuations to the response of an equilibrium system to a weak external perturbation. Specifically, the FDT states that the re- sponse function is proportional to the power spectral density of the thermal fluctuations, with a prefactor given by the temperature. To describe non-equilibrium systems, the idea is to relate the (non-equilibrium) fluctuations to the response function via a (possibly time- or frequencydependent) effective temperature $T_{\text {eff }}[1]$. Violations of the equilibrium FDT have recently been studied extensively in theory and simulations for various model systems such as structural glasses $[2,3]$, spin glasses $[1,4-6]$ and driven systems such as a fluid under shear [7] and aging critical 
systems $[8]$.

The experimental situation, however, is less conclusive. Experiments have been reported on structural glasses [9], spin glasses [10], granular matter [11], hard sphere colloidal glasses $[12,13]$ and the colloidal glass of Laponite [14-16], with sometimes contradictory results.

For the 'soft' systems considered here, the experimental situation is particularly confusing. For hard sphere colloidal fluids there have been two reports on violation of FDT with very different results. Bonn et. al. determined a $T_{\text {eff }}$ that almost reached 100 and depends strongly on frequency [12] whereas a Wang et. al report a constant effective temperature of twice the bath temperature [13].

Laponite is another glassy system for which contradictory results have been reported. Bellon et. al. [14] reported an effective temperature from electrical measurements indicate a strong violation of the FDT in the frequency range 1-40 Hz, whereas the same group did not see deviations for the mechanical measurements in the frequency range 1-20 $\mathrm{Hz}$ [14]. Abou et. al. also reported an effective temperature different from the bath temperature [15]. In contrast to these findings, our simultaneous measurements of displacement fluctuations and mobility using microrheology in the same system show no deviations from the FDT over several decades in frequency $\left(1-10^{4} \mathrm{~Hz}\right)$ and for all aging times: $T_{\text {eff }}=T_{\text {bath }}[16]$. Therefore experimentally the usefulness of the extension of the FDT to non-equilibrium situations and the concept of effective temperature is still a matter of controversy and deserves further investigation. One of the main difficulties seem to be that the direct and simultaneous measurement of the fluctuation correlation function and the and corresponding response function is experimentally rather cumbersome and requires elaborate and high-resolution experimental techniques.

To test the FDT directly, one can use microrheology to measure both the position fluctuations and the complex compliance of a probe particle simultaneously. There are two classes of microrheology (MR) technique: active and passive. In passive MR, one measures the displacement fluctuations of a probe particle: $x(t)$. Then, assuming that the FDT holds, one can obtain the imaginary part of the response function $\alpha^{\prime \prime}(\omega)$ from the power spectrum of thermal fluctuations $\left\langle|x(\omega)|^{2}\right\rangle[17,18]$. Passive MR was first introduced by Mason et. al. [17, 19] and was already then used in non-equilibrium system of hard sphere glasses. Their conclusion was that the complex shear modulus obtained from the passive MR qualitatively to be in agreement with the bulk rheology. In the active method $[16,20,21]$, one directly measures $\alpha(\omega)$, the mechanical response of the probe particle to an applied oscillatory force. Consequently, by comparing the power spectrum of thermal fluctuations $\left\langle|x(\omega)|^{2}\right\rangle$ with the imaginary part of the response function $\alpha^{\prime \prime}(\omega)$, one can obtain the frequency-dependent effective temperature over a wide range of frequencies $\left(0.1 \mathrm{~Hz}-10^{4} \mathrm{~Hz}\right)$ for soft glassy mate- rials from:

$$
\frac{T_{\text {eff }}}{T_{\text {bath }}}=\frac{\omega\left\langle|x(\omega)|^{2}\right\rangle}{2 k_{B} T_{\text {bath }} \alpha_{\text {active }}^{\prime \prime}(\omega)}
$$

If $T_{\text {eff }}=T_{\text {bath }}$, then the fluctuation-dissipation relation is satisfied. The combined use of active and passive MR thus provides a way to directly test the applicability of the FDT in non-equilibrium systems.

In this Letter, in view of the existing controversies in the literature, we test the validity of the FDT using microrheology on hard sphere colloidal glasses and aging colloidal gels and glasses of Laponite. We do not find any deviations from FDT in any of these systems over the entire range of frequencies probed in the experiments.

Experimental. - Laponite XLG suspensions in pure water were prepared as explained in [16]. Samples containing salt were prepared by diluting the Laponite suspensions in pure water and adding a concentrated salt stock solution [22]. Immediately after the preparation of the samples, we mixed in a small fraction, below $10^{-4} \mathrm{vol}$ $\%$, of silica probe beads. This instant defines time zero of the "waiting time". After mixing we infuse the suspension into a sample chamber of about $50 \mu \mathrm{l}$ volume, consisting of a coverslip and a microscope slide separated by spacers made of UV glue with a thickness of about 70 $\mu \mathrm{m}$ and sealed with vacuum grease at the ends to avoid evaporation of sample.Concentrated hard-sphere-like suspensions were prepared by dispersing sterically stabilized PMMA colloids of a diameter of $397 \mathrm{~nm}$ and a polydispersity of $10 \%$ [23] in a nearly refractive-index and densitymatching mixture of cyclohexyl bromide and cis-decalin $(\mathrm{w} / \mathrm{w}=3: 1)$. The values for the density and the refractive index of the solvent mixture are nearly the same as those reported for PMMA $\left(\rho=1.19 \mathrm{~g} / \mathrm{cm}^{3}\right.$ and the index $n=1.49$ [23]). To screen the charges on the colloidal particles, the mixture was saturated with tetrabutyl ammonium bromide (concentration $300 \mu \mathrm{M}$ ). The shear viscosity of the solvent mixture was measured with a rheometer as $\eta_{s}=2.47 \mathrm{mPa} . \mathrm{s}$. As probe particles we used $1.1 \mu \mathrm{m}$ in diameter melamine resin particles (micro-particles $\mathrm{GmbH}$, Germany) with a density $\rho=1.51 \mathrm{~g} / \mathrm{cm}^{3}$ and refractive index $n=1.68$; the high index makes it possible to optically trap them. We prepared the samples by weight fraction, assuming this to be equal to the volume fraction. The suspension was subsequently introduced into a sample chamber and sealed with epoxy glue to avoid evaporation of the sample. After placing the sample chamber in the microscope, we trapped a single bead, moved it to 20-30 $\mu \mathrm{m}$ above the glass surface, and then performed the active and passive MR experiments with it. All experiments were performed at room temperature $\left(21 \pm 1^{\circ} \mathrm{C}\right)$.

The microrheology setup is described in detail elsewhere $[21,24]$. In brief, it consists of two optical tweezers formed by two independent polarized laser beams that are superimposed. One of the lasers is used to drive the oscillations of the trapped particle (drive laser, $\lambda=1064 \mathrm{~nm}$. The 
other one is stationary and is used to detect the position of the particle (probe laser, $\lambda=830 \mathrm{~nm}$ ). Stable trapping is achieved using a high numerical aperture objective lens. The interference signals of the laser beams emerging from the condenser lens (1.4 NA) after passing through the sample are projected onto quadrant photodiodes (QPD).

In the active measurements, the driving laser trap was oscillated through an acousto-optical deflector (AOD) and the output signal from the QPD that detected the probe laser was fed into a lock-in amplifier. Thus we were able to measure the amplitude and phase of signals resulting from driving laser displacements.

We thus measure phase and displacement amplitude of the particle in response to the oscillatory force exerted in the $y$ direction. We oscillated the drive laser with frequency $f=\frac{\omega}{2 \pi}$ and an oscillation amplitude $L(\approx 70$ $\mathrm{nm})$. This displacement results in an oscillatory force of magnitude $\Re\left\{k_{d}[L \exp (-\imath \omega t)-x(t)]\right\}$ on the bead, where $k_{d}$ refers to the trap stiffness of driving laser. Here, $f_{0}=k_{d} L \exp (-\imath \omega t)$ can be interpreted as the apparent oscillatory force exerted by the drive laser. The particle displacement caused by the apparent oscillatory force is denoted as $x_{\omega}(t)$. The apparent response to the sinusoidal force is defined as

$$
<x_{\omega}(t)>=\alpha_{\text {app }}(\omega) f_{0} \exp (-\imath \omega t)
$$

In practice the lock-in amplifier measures the particle displacements at the oscillation frequency, $\left\langle x_{\omega}(t)\right\rangle=$ $\tilde{x} \exp (-\imath \omega t)$. It can be shown that the apparent complex compliance, which includes the influence of the trap, is [21]

$$
\alpha_{\mathrm{app}}=\frac{\tilde{x}}{f_{0}}=\frac{1}{k_{d}-\imath \omega \xi}
$$

where $\xi$ is a friction coefficient reflecting the memory effects in viscoelastic materials. The true response of the embedding material is $\frac{1}{-\imath \omega \xi}$. Therefore the apparent complex compliance must be corrected as $\alpha=\frac{\alpha_{\text {app }}}{1-k_{d} \alpha_{\text {app }}}$.

Analyzing the data in the active method requires knowing the trap stiffness and calibration of the measured amplitude of response to absolute distances. It also requires a correction of the measured amplitude and phase of the response given the response characteristics of the AOD the details of which can be found in [21]. The trap stiffness and the calibration factor for converting voltage to nanometers for both driving laser and probe laser can be found from the spectra of beads in pure solvents [24].

Since the drive laser was relatively strong $(\approx 50-100$ $\mathrm{mW}$ depending on the experiments), there was the possibility that heating effects influence the experiment. Local heating of the solution can lead to a refractive index gradient that can act as an optical lens causing the deflection of the probe laser; the so called photo-thermal lens effect. We tested for such an effect by measuring the AOD response in an our samples in the absence of a bead and found the effect to be negligible (data not shown).

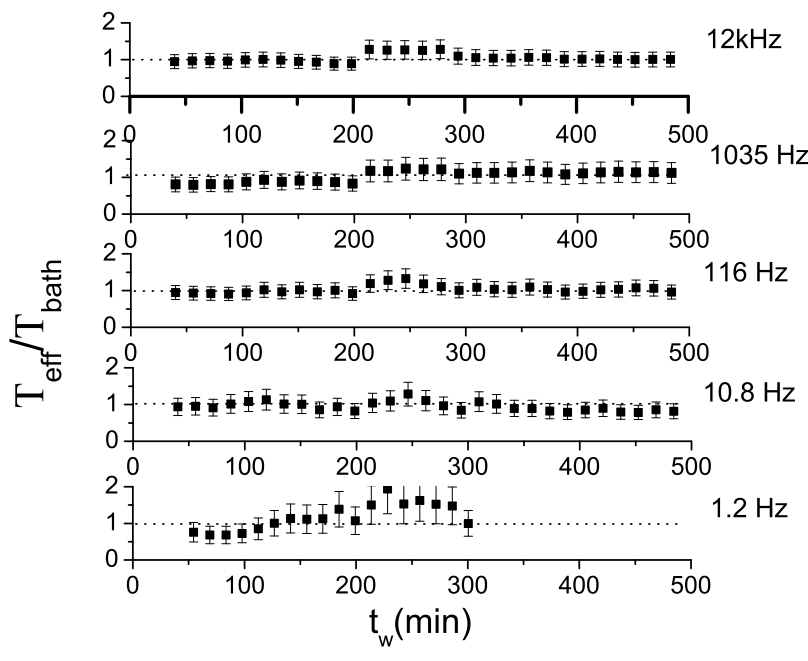

Fig. 1: Effective temperature in Laponite glass $(2.8$ wt\% concentration). Ratio of (apparent) response derived from fluctuations via FDT and actively measured response $T_{\text {eff }} / T_{\text {bath }}$ as a function of waiting time for frequencies $f=$ $12000,1035,116,10.8$ and $1.2 \mathrm{~Hz}$ (from top to bottom). Note that the apparent increase after $200 \mathrm{~min}$, is due to calibration errors, caused by changing the trap power to apply the oscillatory force.

Results. - We have first studied the validity of the FDT in an aging colloidal glass of Laponite, as reported in detail in [16]. We do this by comparing the response functions obtained from active (direct) and passive (indirect) microrheology, where both active and passive measurements are performed on the same bead. The results are plotted in terms of an effective temperature from Eq. (1) in Fig. 1 The figure shows that the effective temperature was equal to the bath temperature to within the experimental uncertainty over a wide range of frequencies $f \approx 1-10^{4} \mathrm{~Hz}$ for all the measured waiting times.

Colloidal gels are another class of non-equilibrium systems showing aging phenomenon. In such systems, similarly to what happens in glasses, correlation and response functions can be a function of time elapsed since their preparation and their relaxation times tend to grow in time. Laponite colloids, within a certain range of colloid concentrations and salt content, can form soft colloidal gels which evolve from an initially liquid-like state to a viscoelastic solid-like state $[26,27]$. The aging mechanism behind the gel formation is different from that of a glass leading to a spatially heterogenous structure in colloidal gels [26-28].

In a recent work [28] we have shown that the heterogenous structure of colloidal gels can be detected by passive MR measurements. Displacement PSDs at intermediate stages of aging measured at almost the same time but different positions of a gel were not equal, hence demonstrating the heterogeneity of the samples at length scales of a few micrometers, contrary to what was found in the glassy samples. Furthermore, at some positions in the gel 
samples we observed some anisotropy, i.e. displacement PSDs measured at $x$ and $y$ directions were not equal.

Despite a number of studies on the validity of FDT in colloidal glasses, there has, as far as we know, as yet been no study investigating the FDT in aging non-equilibrium gels. Here we would like to test the validity of FDT locally at different positions of a gel in spite of the fact that measured PSDs can vary from one point to the other point.

We tested the validity of the FDT in an aging sample of $0.8 \mathrm{wt} \%$ Laponite with $3 \mathrm{mM}$ added salt $\mathrm{NaCl}$ which is known to be in the gel range [27]. To this end, we performed two sets of experiments. First we investigated the FDT at a fixed position, using one and the same probe bead, in the sample at different stages of aging. Similar to the aging colloidal glass, the actively and passively measured responses were in agreement with each other up to the maximum waiting times of $100 \mathrm{~h}$. In a further set of experiments we chose a few beads at different positions in the sample and investigated the validity of the FDT at late stages of aging by performing both active and passive microrheology on each bead in the sample.

Fig. 2] shows the response functions obtained from active and passive microrheology measured at 3 different positions in $0.8 \mathrm{wt} \%$ Laponite (with $3 \mathrm{mM} \mathrm{NaCl}$ ) 5 days after sample preparation. The rate of aging for this sample was, at that time, so slow that there was no significant evolution during one measurement. For beads (a) and (c) the measured PSDs are not equal in the $x$ and $y$ directions. Nevertheless the apparent response from passive microrheology in the $y$ direction agreed well with active response obtained by exerting an oscillatory force in the $y$ direction.

The results show that, despite the presence of heterogeneity in the gels, the FDT in the form of the Einstein relation is valid locally at each point of the sample just as it was for the glassy system.

Next we tested the validity of the FDT in a hard sphere colloidal glass $(\Phi \geq 0.58)$ and a number of supercooled fluids $(0.49<\Phi<0.58)$ as there have also been reports of FDT violation for such systems $[12,13]$. Note that supercooled liquids are in principle ergodic systems and one does therefore not expect to see deviations for the FDT in these systems. However, to resolve the controversy existing in the literature [12], we have done experiments also on such "supercooled" liquids. First, we found that the PSDs of beads measured at different positions in the samples were equal, i.e. that the samples were spatially homogeneous on the micrometer scale. In the measured frequency range, we furthermore did not see any effect of aging (no change of PSDs) in the colloidal glass within the $12 \mathrm{~h} \mathrm{du}-$ ration of the experiments.

As evident in Fig. 3, the agreement between actively and passively measured response functions is excellent. This means again that no deviations from the FDT were observed over the range of frequencies the measurements covered. To extend the range of our measurements to even lower frequencies we have used particle tracking in video microscopy to obtain the PSD for $0.1 \leq \omega<1 \mathrm{~Hz}$. As
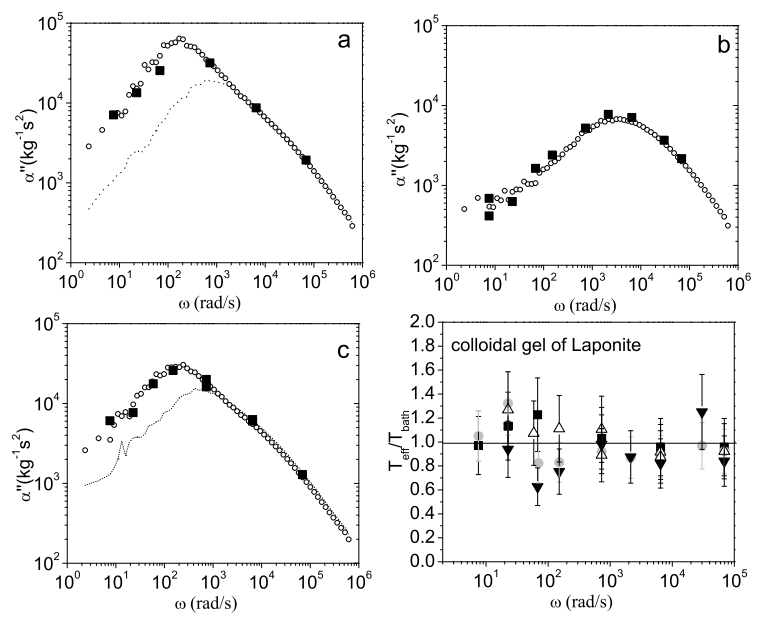

Fig. 2: a,b and c) Imaginary parts $\alpha^{\prime \prime}$ of the frequencydependent response functions in the $y$ direction from active (filled symbols) and passive (open symbols) microrheology using $0.5 \mu \mathrm{m}$ diameter silica beads at 3 different positions of the 0.8 wt\% Laponite $(3 \mathrm{mM} \mathrm{NaCl})$ sample measured 5 days after sample preparation. The dotted lines in panel (a) and (c) show the $\alpha^{\prime \prime}$ in the $x$ direction which is different from its value in the $y$ direction at these positions of the sample. d) Ratio of effective temperature to bath temperature from passive and active MR $T_{\text {eff }} / T_{\text {bath }}$ as a function of frequency, obtained with 0.5 $\mu \mathrm{m}$ diameter silica beads at 4 different positions (shown by the different symbols) in the sample.
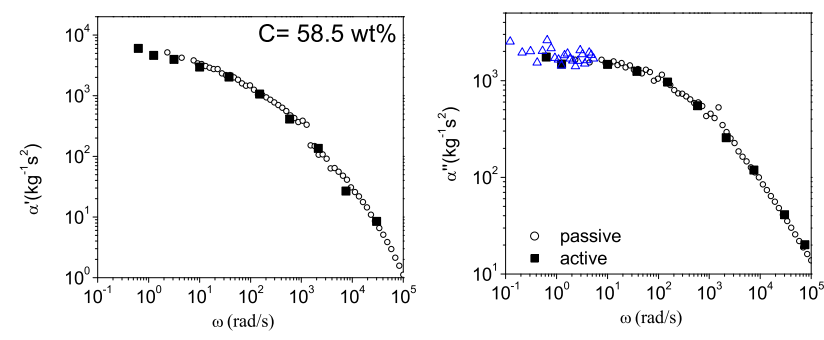

Fig. 3: Real $\alpha^{\prime}$ and imaginary parts $\alpha^{\prime \prime}$ of the frequencydependent response function from active (filled symbols) and passive (open symbols) microrheology using $1.1 \mu \mathrm{m}$ diameter melamine probes for a hard sphere colloidal glass with volume fraction $\phi=0.585$. Data at low frequencies $\omega<10 \mathrm{rad} / \mathrm{s}$ (triangles) were obtained from video microscopy.

can be seen in Fig. 3 there was again a very good agreement between actively and passively measured response.

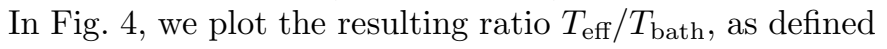
in Eq. (11) as a function of frequency for the two glassy samples and two supercooled liquids. This result confirms again that to within the experimental uncertainty, the FDT is valid in the measured range of frequencies from 0.1 $\mathrm{Hz}$ to $10^{4} \mathrm{~Hz}$ and that the effective temperature does not differ from the bath temperature. This is in agreement with the earlier experiments of Mason for a glassy sample of hard spheres $\phi \approx 0.56$ who compared passive MR 
to bulk rheology over a frequency range $0.1<\omega<100$ $\mathrm{Hz}[19]$.

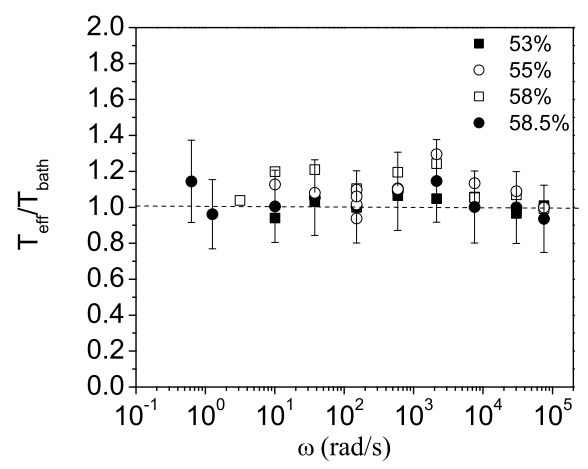

Fig. 4: The effective temperature as a function of frequency for 53 (supercooled fluid), 58 and $58.5 \mathrm{wt} \%$ (glass) $d=397 \mathrm{~nm}$ colloids and 55 wt \% (supercooled fluid) concentration of $d=$ $1.13 \mu \mathrm{m}$ colloids. The concentrations are shown in the legend. The samples in the supercooled region were measured within a couple of hours after preparation, since at later times, we observed the formation of crystals. The glassy samples were measured both immediately after preparation and after $12 \mathrm{~h}$.

Discussion and Conclusions. - We have investigated the validity of the FDT in colloidal glasses and gels of Laponite and in a hard-sphere colloidal glass in the frequency range $0.1-10^{4} \mathrm{~Hz}$. We see in each case a good quantitative agreement between the actively measured response function and the correlation function of spontaneous thermal fluctuations, implying that there is no measurable violation of the FDT in any of these nonequilibrium systems. Equivalently, we find in all cases an effective temperature that does not differ from the bath temperature over the measured frequency range.

Our results disagree with the simulation results which observe deviations from FDT in a binary Lennard-Jones mixed glass [3] and in a fragile glass [2]. In both of these systems the deviations from FDT have been observed at intermediate and long times, when the decay of correlation functions is not exponential anymore. From the former studies [25], it is possible to estimate the frequency range where we should have expected the violation if that ever existed. To do so, we rescale the frequency with the Brownian time scale $\tau_{B}=\frac{R^{2}}{6 D_{0}}$. For our hard sphere colloidal glass with particle radius of $197 \mathrm{~nm}, \tau_{B}=1.46 \times 10^{-2}$ $\mathrm{s}$. Therefore the range of dimensionless frequencies in our measurements vary as $8.7 \times 10^{-3}<\omega \tau_{B}<1.46 \times 10^{3}$. In the data of van Megen et al. [25] for a colloidal glass similar to ours, the plateau region of the intermediate scattering function was observed for time scales $1<t / \tau_{B}<10^{5}$ which corresponds to $6 \times 10^{-5}<\omega \tau_{B}<6$. Therefore at least part of the frequency range of our measurements is in the plateau region corresponding to the time regime for which the models suggest that the FDT violations should be visible. Besides the obvious difference between such 'real' glasses and soft glasses considered here, we have no explanation for this discrepancy.

The same experimental systems as those studied here had been investigated by other groups, as we mentioned in the introduction. The rheological measurements of the Ciliberto group in a colloidal glass of Laponite are in agreement with our results whereas their electrical measurements disagree [14]. The reason for the disagreement could be that in the electrical measurements a different degree of freedom was monitored. In contrast to this, simulations on a sheared binary Lennard-Jones fluid [7] have shown that the effective temperature are independent of the chosen observable in such systems. To confuse matters even more, it has been shown theoretically [5] that the effective temperature in the glass phase of the Bouchaud's trap model does depend on the observable. Subsequent work of Cilibetro et al on the Laponite [14] (2003) showed, however, that the observed violations of electrical FDT were due to violent and intermittent events with unknown origin.

Abou et al found violations of FDT during the aging of Laponite system by using an experimetnal approach similar to ours [15]. Our method, however, is more direct, since we are measuring the fluctuations and the response with the very same probe particle.

In hard sphere glasses there have also been reports of violations of the FDT. The large violations reported by Bonn and Kegel [12] are likely to be due to the fact that they compared rheological measurements and light scattering experiments from two different systems, although both were in principle hard sphere systems. Furthermore, they did not use the correct form of Stokes formula in their analysis. The Makse group [13] has reported an effective temperature for a hard sphere glass $(R=1.5 \mu \mathrm{m})$ that was twice the bath temperature at long times $t \sim 1000 \mathrm{~s}$. This time corresponds to a dimensionless frequency $\omega \tau_{B} \approx 4 \times 10^{-2}$, which is comparable to our lowest measured frequencies $\omega \tau_{B} \approx 10^{-2}$. In [13], mobility was determined as the ratio of a constant force to the response displacement of a particle, which is not generally accepted way to estimate the effective temperature (especially for glassy materials taking infinite time to relax).

Recently Greinert et al. [29] also have reported a $T_{\text {eff }}$ larger than bath temperature for an aging colloidal glass of Laponite at late stages of aging. Although their method using equipartition theorem rather than FDT might be useful to investigate the nonequilibrium signature at low frequencies, technically it is difficult to exactly rule out the low fequency noise from the displacement fluctuation, which leads to apparent $T_{\text {eff }}$ higher than $T_{\text {bath }}$. In fact Jop. et al. have performed measurements using the same method as Greinert et al. and find an effective temperature equal to bath temperature [30], suggesting that inferring an effective temperature necessitates a careful treatment of data at long waiting times.

In conclusion, we have here applied a technique that offers a direct method for the simultaneous measurement 
of response and correlation functions. This overcomes difficulties for experiments with non-equilibrium soft materials or glasses which have hampered earlier approaches searching for an effective temperature different from the ambient temperature. Our experiments on glasses, gels and supercooled liquids show no deviations from FDT.

$$
* * *
$$

This research was supported by the Foundation for Fundamental Research on Matter (FOM) of the Dutch NWO. LPS de l'ENS is UMR8550 of the CNRS, associated with university Paris 6 and 7. Further support (C.F.S.) came from the DFG Center for the Molecular Physiology of the Brain (CMPB) and the Sonderforschungsbereich 755 of the German Science Foundation (DFG).

\section{REFERENCES}

[1] L. Cugliandolo, J. Kurchan, and L. Peliti, Phys. Rev. E 55, 3898 (1997).

[2] G. Parisi, Phys. Rev. Lett. 79, 3660 (1997).

[3] W. Kob and J. L. Barrat, Europhys. Lett. 46, 5 (1999).

[4] L. F. Cugliandolo and J. Kurchan, Phys. Rev. Lett. 71, 173 (1993).

[5] S. M. Fielding and P. Sollich, Phys. Rev. E 67, 011101 (2003).

[6] T. M. Nieuwenhuizen, Phys. Rev. Lett. 80, 5580 (1998).

[7] L. Berthier and J. Barrat, Phys. Rev. E 63, 012503 (2000); L. Berthier and J. L. Barrat, The Journal of Chemical Physics 116, 6228 (2002); N. Xu and C. S. O?Hern, Phys. Rev. Lett. 94, 055701 (2005).

[8] P. Calabrese and A. Gambassi, J. Phys. A: Math. Gen. 38, R133 (2005); J. Stat. Mech.: Theory and Experiment 2004, P07013 (2004)

[9] T. S. Grigera and N. E. Israeloff, Phys. Rev. Lett. 83, 5038 (1999).

[10] D. Hérisson and M. Ocio, Phys. Rev. Lett. 88, 257202 (2002).

[11] P. W. C. M. Song and H. A. Makse, Proc. Nat. Acad. Sci. U.S.A 102, 2299 (2005); G. D'Anna, P. Mayor, A. Barrat, V. Loreto, and F. Nori, Nature 424, 909 (2003).

[12] D. Bonn and W. K. Kegel, J. Chem. Phys. 118, 2005 (2003).

[13] P. Wang, C. M. Song, and H. A. Makse, Nat. Phys. 2, 526 (2006).

[14] L. Bellon, S. Ciliberto, C. Laroche, Europhys. Lett. 53, 511 (2001); L. Bellon, S. Ciliberto, Physica D 168-169, 325 (2002); L. Buisson, L. Bellon, and S. Ciliberto, J. Phys. Cond. Matt. 15, S1163 (2003).

[15] B. Abou and F. Gallet, Phys. Rev. Lett. 93, 160603 (2004).

[16] S. Jabbari-Farouji, D. Mizuno, M. Atakhorrami, F.C. MacKintosh, C.F. Schmidt, E. Eiser, G.H. Wegdam and D. Bonn,Phys. Rev. Lett. 98, 108302 (2007).

[17] T.G. Mason, and D.A. Weitz, Phys. Rev. Lett. 74, 1250 (1995).

[18] F. Gittes, B. Schnurr, B. and Olmsted, P.D. F.C. MacKintosh, and C.F. Schmidt, Phys. Rev. Lett. 79, 3286 (1997); B. Schnurr, F. Gittes, F.C. MacKintosh, C.F. Schmidt, Macromolecules 30, 7781 (1997).
[19] T.G. Mason, H. Gang and D.A. Weitz J. Opt. Soc. Amer. A 14 (1997) 139.

[20] L. A. Hough and H. D. Ou-Yang, Phys. Rev. E 65, 021906 3 (2002).

[21] D. Mizuno, C. Tardin, C. F. Schmidt and F. C. MacKintosh Science 315, 370 (2007); D. Mizuno et. al. in preparation.

[22] Nicolai and S. Cocard, Langmuir 16, 8189 (2000).

[23] R. P. A. Dullens, M. Claesson, D. Derks, A. van Blaaderen, and W. K. Kegel, Langmuir 19(15), 5963, 2003.

[24] M. Atakhorrami, J. Kwiecinska, K. M. Addas, G. H. Koenderink, A. Levine, F. MacKintosh, and C.F. Schmidt, Phys. Rev. E 73 (2006), part 1061501.

[25] W. van Megen, T. C. Mortensen and S. R. Williams, Phys. Rev. E 58, 6073 (1998).

[26] S. Jabbari-Farouji, G. H. Wegdam and D. Bonn, Phys. Rev. Lett. 99, 065701 (2007).

[27] S. Jabbari-Farouji, H. Tanaka, G. H. Wegdam and D. Bonn, Multiple nonergodic disordered states in Laponite suspensions: a phase diagram, arXiv:0804.3330.

[28] S. Jabbari-Farouji, M. Atakhorrami, D. Mizuno, E. Eiser, G.H. Wegdam, F.C. MacKintosh, D. Bonn and C.F. Schmidt, arXiv:0710.5459, submitted to Phys. Rev. E.

[29] N. Greinert, T. Wood, P. Bartlett, Phys. Rev. Lett. 97 265702 (2006).

[30] P. Jop, A. Petrosyan and S. Ciliberto, Comment on Measurement of Effective Temperatures in an Aging Colloidal Glass, arXiv:0705.1421 v1. 